\title{
Mindfulness for the management of cachectic cancer patients: A randomized study
}

\author{
Christian Focan $^{1 *}$, Ghislain Houbiers ${ }^{1}$, Laura Gilles ${ }^{2}$, Vincent Baro ${ }^{3}$ and Marie-Pascale Graas ${ }^{1}$ \\ ${ }^{1}$ Department of Oncology, CHC-Liège, Belgium \\ ${ }^{2}$ Department of Dietetics, CHC-Liège, Belgium \\ ${ }^{3}$ Department of Psychology, CHC-Liège, Belgium
}

Cancer cachexia is a complex syndrome characterized not only by a significant weight loss with rapid fall of body mass index (BMI), fatigue, drop of performance clinical indices but also by an important muscle wasting with loss of lean body mass and physical power, and biological abnormalities such as inflammatory syndrome, anemia, reduction of albumin and pre-albumin serum rates [1]. Cachexia, observed in more than $50 \%$ of cancer cases, is not easy to manage and, by now, no standard treatment has been acknowledged [1].

Recently, some teams have proposed to explore possibilities of complementary medicine, such as physical exercise and therapies from oriental inspiration (Tai-chi; Qi-gong). Also some psychiatric groups have studied the ability to help patients in the relief of their stress by applying meditation tools as full body mindfulness [2].

To the best of our knowledge however, no team has proposed to cancer patients to participate to workshops specifically structured on the concept of mindfulness and developed not only on a psychological dimension as previously proposed by some authors [2] but also for the first time on a dietetic dimension. This type of management has been implemented by our group in a randomized study held as a pilotproject having benefited from a grant from the Cancer-Plan Belgium.

The study protocol received approval from Clinique SaintJoseph (Liège; Belgium) ethics committee on June $12^{\text {th }} 2012$ (study $11 / 22 / 597)$. Cancer patients treated in our oncology department developing evidence of cachexia [1] were proposed, after informed consent, to be included in a randomized trial aiming to compare standard management(control group B) versus standard management and voluntary participation (experimental group A) to psychological and dietetics workshops offering a cognitivo-behavioural approach based on full body mindfulness philosophy. Four double workshops were programmed every 2 weeks. Details on the coverage proposed in these dietary and psychological specialized workshops are available on request (christian.focan@chc.be).

All subjects benefited from the same clinical and biological evaluation as well as from the same detailed quantitative and qualitative food anamnesis (daily ingested total calories and distribution of calorie intakes in carbohydrates/lipids/proteins). These assessments were proposed before the beginning of workshops (time 0 ; T0), one month later or after $2^{\star} 2$ workshops (T 1 month; T1), and 2 months later or after $4 \times 2$ workshops (T 2 months; T2).

Furthermore, questionnaires aiming to evaluate quality of life and psychological mindfulness perception were administered to all patients at the 3 experimental times. For quality of life, the EORTC-
QLQ-C30 was elected [3] while for mindfulness approach, the FFMQ (Five facet Mindfulness Questionnaire) validated in various languages (ie in french one) was applied [4]. EORTC questionnaire comprises 30 randomly distributed questions (with quotation from 0 to 4 ) allowing after grouping to obtain scoring for global health, physical, emotional, cognitive and social functioning; also some scorings are centered on symptoms (fatigue; pain; sleep disturbances; appetite) and treatments side-effects (GI-tract) [3]. In FFMQ document, 40 questions quoted from 1 to 5 (scale ranging from 'never or very rarely true' to 'very often or always true') also randomly applied, allowed after grouping to assess psychological behaviour in terms of observation, description, action, no judgment and lack of reactivity [4]. Data collected by both questionnaires for the aim of a comparative analysis had to be added, normalized (standardized) and directed so that the higher the value between 0.0 and $100.0 \%$ (thus inversing scoring for some questions), the more positive for the patient was the estimated variable $[3,4]$.

Data were statistically compared at the 3 experimental times (T0, $\mathrm{T} 1$ month, and T 2 months). All analyses were processed through the software SAS v9.2. Continuous data were compared by Student-T, Wilcoxon and Kuskall-Wallis non-parametric tests. Categorical data were compared by chi-square and exact Fisher tests.

Between November 2011 and july 2013, 551 patients were screened regarding a possible cachexia. Among them, 429 (77.8\%) were fulfilling inclusion criteria. However only 53 subjects (group A: 27 subjects and group B: 26 subjects) finally accepted to participate to the trial. Reasons for refusal were multiple: lack of personal interest $(225 ; 52.4 \%)$, weakness $(44 ; 10.3 \%)$, transport problems $(28 ; 6.5 \%)$, early deaths $(62$; $14.5 \%)$, and medical refusal (17; 3.9\%).

At inclusion time, no statistical difference appeared between groups $\mathrm{A}$ and $\mathrm{B}$ on patient characteristics via clinical and biological evaluations. The majority of patients suffered from metastatic solid tumors (92.6\% group A vs $96.2 \%$ group B). Control B group had more grades 2 and 3 anorexia $(\mathrm{p}=0.002)$ and a trend to lower rate of metastases (85.2\% group A vs $65.4 \%$ group $B ; p=0.06$ ). Furthermore, no difference in initial quantitative or qualitative calories intakes was

Correspondence to: Christian Focan, $\mathrm{MD}, \mathrm{PhD}, \mathrm{CHC}$, Clinique Saint-Joseph, 75, rue de Hesbaye, B-4000-LIEGE, Belgium, Tel: +32-4-2248990 ; Fax: +32-42248991; E-mail: christian.focan@chc.be

Keywords: cancer, cachexia, dietetics, mindfulness

Received: April 02, 2016; Accepted: April 14, 2016; Published: April 18, 2016 
noticed between both groups ( $\mathrm{p}>0.05)$.

No difference was observed between data recorded at experimental times $\mathrm{T} 1$ and $\mathrm{T} 2(\mathrm{p}>0.05)$.

Subjects participating to workshops (group A) ultimately had a favorable evolution regarding weight $(\mathrm{p}<0.01), \mathrm{BMI}(\mathrm{p}=0.04)$, and clinical WHO/ECOG indices [5] $(\mathrm{p}=0.004)$ (Table 1). On the contrary, no difference could be detected (either between groups $\mathrm{A}$ and $\mathrm{B}$ or insides groups at T0 vs T1/T2 levels) with regards to biological parameters, quantitative, relative qualitative calories intakes, and nutritional indices (NRS) ( $\mathrm{p}>0.05)$ [6]. All recorded and normalized data obtained by the 2 questionnaires (EORTC and FFMQ) were compared as described above. The only variables significantly improved were observed in the experimental group, thus emotional function, fatigue and given digestive disturbances (nausea; vomiting; constipation) (questionnaire EORTC) as well as faculty of observation (questionnaire FFMQ) $(\mathrm{p}<0.05)$.

The aim of our study was to allow cachectic cancer patients to benefit from the mindfulness concept applied both at the psychological level and at the dietary level in dedicated workshops. To the best of our knowledge, this original pluridisciplinary management had never been reported in the literature. At the dietary level, patients were invited to evaluate various aliments by appealing to their 5 senses. The mindfulness psychological method is a cognitivo-behavioural approach inspired from buddhism philosophy which leads patients to concentrate on the actual moment making abstraction from any other temporal contingency or from any other worrisome problem. By an intense concentration on the actual moment, one can live fully any instant without judging on experience spreading out minute by minute. Mindfulness indicates the quality of consciousness which emerges when one turns the spirit at about the present moment. Patients can find by this way some means to better answer to their needs, to better stay at the listening of their body, to live better general discomfort, to relieve stress and even to expose themselves to the fear of a recurrence of the disease [2]. Its contribution for the management of cancer patients has been recently acknowledged $[2,7,8]$. It has been

Table 1. Weight, BMI and clinical indices evolution.

\begin{tabular}{|c|c|c|c|}
\hline Variable & Group A & Group B & p-value \\
\hline Mean variation $(\mathrm{kg})$ & +1.32 & -1.47 & \multirow[t]{2}{*}{0.01} \\
\hline Limits $(\mathrm{kg})$ & $-1.4-+5.8$ & $-10-+1.0$ & \\
\hline \multicolumn{4}{|l|}{ BMI } \\
\hline Mean variation (weight $/$ size $^{2}$ ) & +0.31 & -0.57 & \multirow[t]{2}{*}{0.04} \\
\hline Limits & $-1.1-+1.2$ & $-4.8-+2.4$ & \\
\hline \multicolumn{4}{|l|}{ WHO scores } \\
\hline$\%$ improvement & 57.1 & 5.5 & 0.004 \\
\hline
\end{tabular}

No difference was observed between $\mathrm{T} 1$ and $\mathrm{T} 2$ assessment times occasionally tested in randomized studies in comparison to more traditional initiatives, with positive outcome expressed on stress reduction, positive psychological outcomes, symptoms burden and some biomarkers $[7,8]$. This was evidenced especially within cancer patients in search of meaning [8]. However, by now, it had never been associated to a dietary appropriated and adapted dimension, especially for cancer patients groups in peculiar difficulty, such as those suffering from evolutive cachexia.

Despite difficulties in terms of feasibility as well as of recruitment (with only $12.3 \%$ of candidates having finally accepted to participate) or of general compliance to workshops, our randomized trial has shown that it was possible to allow small groups of cachectic cancer patients to benefit from psychological and dietary mindfulness workshops. Subjects from the experimental group, though presenting at initial assessment with less favorable clinical items (trend to more frequent metastases and more important anorexia), were globally satisfied from their peculiar management. They could enjoy significant weight gain and had an evident improvement of their general status. Also their quality of life was improved as regards emotional function, observation faculty, fatigue and digestive troubles.

For the future, this type of specialized management could only be proposed to especially motivated patients with neoplastic cachexia.

\section{Conflict of interest}

The authors have nothing to disclose and indicated no potential conflict of interest.

\section{References}

1. Tuca A, Jimenez-Fonseca P, Gascón P (2013) Clinical evaluation and optimal management of cancer cachexia. Crit Rev Oncol Hematol 88: 625-636. [Crossref]

2. Gotink RA, Chu P, Busschbach JJ, Benson H, Fricchione GL, et al. (2015) Standardised mindfulness-based interventions in healthcare: an overview of systematic reviews and meta-analyses of RCTs. PLoS One 10: e0124344. [Crossref]

3. EORTC -QLQ-C30 scoring manual. The EORTC QLQ-C30 (2001) Third edition. pp 78.

4. Heeren A, Douilliez C, Peschard V, Debrauwere L, Philippot P (2011). Cross-cultural validity of the Five Facets Mindfulness Questionnaire: adaptation and validation in a French-speaking sample. Revue européenne de psychologie appliquée 61: 147-51.

5. Buccheri G, Ferrigno D, Tamburini M (1996) Karnofsky and ECOG performance status scoring in lung cancer: a prospective, longitudinal study of 536 patients from a single institution. Europ J Cancer 32A: 1135-41.

6. Cereda E, Limonta D, Pusani CD, Vanotti A (2006) Geriatric nutritional risk index: a possible indicator of short-time mortality in acutely hospitalized older people. $J \mathrm{Am}$ Geriatr Soc 54: 1011-12.

7. Zhang MF, Wen YS, Liu WY, Peng LF, Wu XD, et al. (2015) Effectiveness of Mindfulness-based Therapy for Reducing Anxiety and Depression in Patients With Cancer: A Meta-analysis. Medicine (Baltimore) 94: e0897-0890. [Crossref]

8. Rouleau CR, Garland SN, Carlson LE (2015) The impact of mindfulness-based interventions on symptom burden, positive psychological outcomes and biomarkers in cancer patients. Cancer Manag Res 7: 121-31.

Copyright: (C2016 Focan C. This is an open-access article distributed under the terms of the Creative Commons Attribution License, which permits unrestricted use, distribution, and reproduction in any medium, provided the original author and source are credited. 